treatment efficacy. It also highlights the sub-optimal efficacies of emerging treatments currently in clinical trial to treat extragenital gonorrhoeal infections and suggest optimisation during clinical trials could potentially improve the efficacy of these treatments to reach the marketplace while balancing patients side effects.

\section{SO2.3 MOLECULAR TESTING FOR STI ANTIMICROBIAL RESISTANCE: TACKLING A MOVING TARGET}

D Whiley*. The University of Queensland, Brisbane, Australia

10.1136/sextrans-2021-sti.24

The gonococcus is now presenting some serious public health and clinical challenges. Notably, antibiotic-resistant Neisseria gonorrhoeae strains continue to emerge and spread and now threaten key gonorrhoea treatments, including ceftriaxone. Alternate treatment strategies are needed. Individualized therapy, whereby a rapid resistance test is used to inform individual patient treatment, is one proposed strategy. The use of individualised therapy in now already embedded in treatment of antibiotic (azithromycin)-resistant Mycoplasma genitalium infection, but is still in its infancy for the gonococcus. Key challenges exist, including knowledge gaps relating resistance mechanisms, the fact that sequence targets may change over time. These hurdles are not insurmountable, and can potentially be overcome via enhanced international collaboration and sequence/data sharing.

\section{SO2.4 CONCORDANCE BETWEEN GENOTYPIC AND PHENOTYPIC RESISTANCE, AND IMPLICATIONS FOR SURVEILLANCE OF AMR}

M Cole*. Public Health England, UK

10.1136/sextrans-2021-sti.25

Culture-based phenotypic antimicrobial susceptibility testing (AST) is the gold-standard method for determining Neisseria gonorrhoeae antimicrobial resistance (AMR). AMR surveillance can be enhanced by the molecular detection of resistance directly from clinical specimens, usually by nucleic-acid amplification techniques (NAATs). AMR surveillance is then possible in areas that struggle with obtaining viable cultures. NAAT AMR detection can be more sensitive and quicker than culture-based AST and can subsequently improve sample size and representativeness. Available NAATs do not however reliably detect all resistance markers, so some selection is required.

Whole-genome sequencing (WGS) is normally performed using cultured isolates and this enables detection of all known resistance markers. If the sequence is linked to culture, then the data can be used to detect unknown resistance determinants. Additional advantages of using WGS for AMR surveillance activities include the identification of AMR clones and transmission networks to further enhance $\mathrm{N}$. gonorrhoeae molecular epidemiology.

Genotypic and phenotypic resistance concordance is high for some antimicrobials such as ciprofloxacin, so using genotypic, in place of phenotypic methods, can be done with confidence. For some antimicrobials, such as cefixime and ceftriaxone, resistance can be multi-factorial so multiple loci detection/sequencing and appropriate algorithms need to be applied to predict resistance. Even then, the predicted resistance may not always correlate with the MIC. Azithromycin can also be a challenge as the exact mechanisms of resistance have not been identified for all isolates.

As genotypic methods alone cannot detect the emergence of novel resistance and not all resistance mechanisms are known, then appropriate sampling would be required if NAATs or WGS were to be solely used in surveillance studies. It is likely that AST via phenotypic methods has a role in AMR surveillance for some time to come, but AMR surveillance would certainly be enhanced by genotypic resistance detection.

\section{S03.1 ROLE OF MYCOPLASMA GENITALIUM IN SYMPTOMS AND SYNDROMES IN CISGENDER WOMEN}

M Trent*. Johns Hopkins Schools of Medicine and Public Health, Baltimore, Maryland, USA

\subsection{6/sextrans-2021-sti.26}

Mycoplasma genitalium (MG) has been a known pathogen affecting reproductive health outcomes since the 1980s. However, it was not officially considered in the Centers for Disease Control and Prevention (CDC) STI treatment guidelines until 2015. The paucity of population-based epidemiological data and clinical outcomes data from randomized controlled trials limits the scope of the CDC recommendations. While increased attention to $\mathrm{MG}$ as a pathogen has been driven by complicated presentations of urethritis in cisgender males, MG also has significant implications for sexual and reproductive health outcomes in cisgender women, including maternal-child health outcomes. Recurrent and persistent infection may be associated with ongoing inflammation in the female genital tract that may increase secondary STI and HIV infection risk due to resulting shifts in vagina microbiota. While there are no public health control programs in the United States for MG, several other countries have recognized the importance of prioritizing MG as a public health issue. In this session, we will examine MG in the context of adolescent and young adult cisgender women's health disparities and discuss the potential for strategic interventions in practice.

\section{S03.2 WHAT IS THE ROLE OF QUINOLONE RESISTANCE TESTING IN THE MANAGEMENT OF M. GENITALIUM?}

G Murray*. Royal Women's Hospital, Melbourne, Australia

\subsection{6/sextrans-2021-sti.27}

In the wake of escalating macrolide resistance, resistance to fluoroquinolones has emerged as a problem requiring urgent attention. The mechanisms of resistance for macrolides (single nucleotide mutations in the $23 \mathrm{~S}$ rRNA gene) are well defined both in vitro and clinically through analysis of treatment failure; resistance-guided therapy based on these mutations can achieve first line cure levels of over 90\%. This contrasts with our understanding of the mechansims of fluoroquinolone resistance, where multiple single nucleotide polymorphisms in the parC gene have been postulated to contribute, but evidence supporting a role of each mutation is limited. Currently 